\title{
Biological parameters affecting transient GUS gene expression in oil palm (Elaeis guineensis Jacq.) embryogenic calli via microprojectile bombardment
}

\begin{abstract}
Biological parameters affecting microprojectile bombardment delivery of DNA into oil palm embryogenic calli were optimized by monitoring transient GUS gene expression. The parameters optimized were the following; explant type using gold and tungsten microcarrier, bombardment preculture, time between bombardment and GUS staining, genotype, immature embryo preculture, DNA concentration, osmoticum type and concentration, and osmoticum treatment duration before and after bombardment. An independent experiment was carried out to study the effect of each parameter and its variables on transient GUS expression. ANOVA was carried out for each experiment using completely randomized design and the treatment means were compared using Duncan's Multiple Range Test. The highest transient GUS expression was observed 2 days post-bombardment using embryogenic calli derived from immature embryos. Bombardment was carried out using $300 \mathrm{gg}$ of gold microcarriers coated with 1.5 हg of DNA $24 \mathrm{~h}$ after transfer to fresh medium. GUS expression could be further enhanced when calli were transferred to medium containing osmoticum $(0.4 \mathrm{M}$ mannitol) $2 \mathrm{~h}$ prior to bombardment. Highly significant differences between the variables were observed for all the parameters studied except genotype.
\end{abstract}

Keyword: Microprojectile bombardment; Oil palm; Optimization; Biological parameters 